\title{
Removing Artifacts from EMG Envelope for Improving Myoelectric Robot Arm Control
}

\author{
SANDRA M'ARQUEZ-FIGUEROA \\ Dept. of Electronics Engineering \\ Universidad de Guanajuato \\ Salamanca, MEXICO
}

\author{
YURIY S. SHMALIY \\ Dept. of Electronics Engineering \\ Universidad de Guanajuato \\ Salamanca, MEXICO
}

\author{
OSCAR IBARRA-MANZANO \\ Dept. of Electronics Engineering \\ Universidad de Guanajuato \\ Salamanca, MEXICO
}

\begin{abstract}
There are analysis methods of biomedical signalfeatures at present to know any information regarding the humanbody. It is to use the extracted features from the EMG signalto predict human motion and its associated efforts by usingsignals given by the motor unit action. Steps to process theEMG signal are envelope acquiring, artifacts filtering, estimationsmoothing, EMG value standardizing, feature classifying, andmotion recognizing. Different methods are useful to achieve thisgoal and apply by experimental projects. Using a database ofEMG signals, we calculate the envelope by using the rectifiedsignal, where we take the absolute number of EMG signals so thatall values become positive. In the first step, we shall now proceedto remove EMG envelope artifacts by using filters such as theKalman filter (KF), H1 filter, unbiased finite impulse response (UFIR), cKF, cH1, and cUFIR. The last three algorithms wereamended by assuming colored measurement noise. Last, we makea standardization of the EMG envelope. Given the above, we willknow if the estimation envelope gives the optimal features for anaccurate prediction.
\end{abstract}

Key-Words: EMG signal envelope, filtering, features, classification, outliers

Received: July 27, 2019. Revised: January 27, 2020. Accepted: February 16, 2020. Published: February $28,2020$.

\section{Introduction}

Experimental investigations have been carried out for diverse Electromyography(EMG) raw databases to show better signal features. It tested by several standard metrics and different methods [1]. EMG signals are a register of the electrical activity of the muscles with positive and negative deflections. Methods of classification analyze the performance of the obtained data from the EMG signal and can be combined with different technologies for a better result to identify the information. To effectively do that, it is necessary to remove the noise which figures as an undesired component in the EMG record by using good quality filter digital [2], [3]. It applies in bio-electronics [4], bio-mechanics [5], [6], and bio-robotics [7], where use EMG features such as frequency and amplitude to identify motions.

EMG envelope is getting by using the rectified signal, where the value negative becomes positive [8], [9]. Some research uses the reflected envelope and achieves an efficient noise reduction. But, it produces unacceptable bias errors [10], [11]. However, the EMG envelope does not prevent spikes . Thus, It is necessary to use filter technics. Thus, It is necessary to use filter technics. In [12] improves the envelope by using the Savitsky-Golay smoother combined with a low-pass filter. But, it introduces time-delay-lags. However, it introduces timedelay-lags. In this investigation, we use the developed filters in [3], where high accuracy is demonstrated for removing artifacts from the EMG envelope. We employ the NinaPro database, which contains the records of the upper limbs of 27 intact subjects while performing 52 fingers, hand, and wrist movements of interest [13]. The data resolution over a $10 \mathrm{mV}$ range. All tests of synthetic data are provided by using special

This investigation was partly supported by the Mexican CONACyT-SEP Project A1-S-10287, Funding CB2017-2018. software. The value of noise indicators can be determined either by measurement or computation [14]-[17].

\section{EMG Signal Envelope and Problem Formulation}

\subsection{EMG Signal Envelope}

We need to get the EMG envelope due to EMG is a bipolar signal meaning that it goes into both positive and negative direction and has a base line around zero, the rectification signal is applied to get the absolute number of EMG therefore after the rectification all values become positive however we can notice the rectified EMG still has a lot of spikes. these spiks will be eliminated by using smoothing algorithms. For this, we can approximate the EMG envelope to a polynomial by using the Taylor series expansion as 11 , which is a way of approximating any function by a polynomial [18],

$$
F=\left[\begin{array}{ccccc}
1 & \tau & \frac{\tau^{2}}{2} & \cdots & \frac{\tau^{K-1}}{(K-1) !} \\
0 & 1 & \tau & \cdots & \frac{\tau^{K-2}}{(K-2) !} \\
0 & 0 & 1 & \cdots & \frac{\tau^{K-3}}{(K-3) !} \\
\vdots & \vdots & \vdots & \ddots & \vdots \\
0 & 0 & 0 & \cdots & 1
\end{array}\right] \in \mathbb{R}^{K \times K},
$$

where $K$ is the number of the envelope states and $F$ is the system matrix.

The problem focuses on identifying the best processing algorithm achieving the highest estimation, in order to increase the accuracy of the features. Therefore, we make a comparison between traditional filters and modified filters assuming CMN using advanced statistical techniques. Thus, our report can be better geared towards the monitoring and evaluation of its 
trends. In addition, we use two additional algorithms to apply smoothing to the estimate of each filter.

\subsection{Kinematic Model of Robot Arm}

Dynamic systems are understood when we have adequate knowledge of their functioning. We are going to review the structure of a robotic arm, it constitutes of links, joints, and handlings, where the inclination angle and the length of the first and second link are given by $\Phi_{1}, l_{1}, \Phi_{2}$, and $l_{2}$. The system will recognize the EMG motion with an accurate to the millimeter. The kinematic model of a robotic arm gives the related speed $\dot{h}_{(X, Z)}$ of an interesting point to the angular speed $\dot{\Phi}$ for each motor located in the joints. We get this relationship by using the Jacobian matrix. To obtain the mathematical model of a robotic arm with two degrees of freedom (2DOF), we consider, is defined by the plane perpendicular to the reference $X, Z$. From the Creeper we create the mathical model.

$$
\begin{aligned}
h_{X}= & X_{1}+X_{2}, \\
h_{Z}= & b+Z_{1}+Z_{2}, \\
h_{Z}= & b+Z_{1}, \\
h_{X}= & l_{1} \cos \left(\Phi_{1}\right)+l_{2} \cos \left(\Phi_{1}+\Phi_{2}\right), \\
h_{Z}= & b+l_{1} \sin \left(\Phi_{1}\right)+l_{2} \sin \left(\Phi_{1}+\Phi_{2}\right), \\
\dot{h}_{X}= & \left(-l_{1} \sin \left(\Phi_{1}\right)-l_{2} \sin \left(\Phi_{1}+\Phi_{2}\right)\right) \dot{\Phi_{1}} \\
& -\left(l_{2} \sin \left(\Phi_{1}+\Phi_{2}\right)\right) \dot{\Phi_{2}}, \\
\dot{h}_{Z}= & \left(l_{1} \cos \left(\Phi_{1}\right)-l_{2} \cos \left(\Phi_{1}+\Phi_{2}\right)\right) \dot{\Phi_{1}} \\
& +\left(l_{2} \cos \left(\Phi_{1}+\Phi_{2}\right)\right) \dot{\Phi_{2}},
\end{aligned}
$$

where $\Phi_{1}$ and $\Phi_{2}$ are the angle of inclination of each link Kudo, $l_{1}$ and $l_{2}$ are the lengths of each of the links, $\dot{h_{x}}$ and $\dot{h}_{y}$ are the angular speeds, $\dot{\Phi}_{1}$ and $\dot{\Phi_{2}}$ are the speeds of each the motors located in the links, while $\ddot{\Phi}_{1}$ and $\ddot{\Phi}_{2}$ give the accelerations. This, in order to control the motor position according to the reference position and thus be able to bring the application to control the motors of a prosthesis. to prove it, equations of the dynamic system can be expressed as a mathematical model with a set of variable inputs.

\section{Materials and Methods}

\subsection{State-space Model of EMG Envelope}

The EMG envelope is represented in discrete-time index $i$ with the $k$-state-space polynomial model [18] assuming GaussMarkov CMN $v_{i}$ as [19]

$$
\begin{aligned}
x_{i} & =F x_{i-1}+B w_{i}, \\
y_{i} & =H x_{i}+v_{i}, \\
v_{i} & =\psi_{i} v_{i-1}+\xi_{i},
\end{aligned}
$$

where

- $x_{i}$ is the state vector.

- $y_{i}$ is the scalar observation of the envelope first state.

- $H$ is the observation matrix.
- $\psi_{i}$ is the scalar coloredness factor.

- $B$ is the system input matrix. $w_{i}$ is the envelope noise.

The matrix observation is expressed as $H=\left[\begin{array}{llll}1 & 0 & \ldots\end{array}\right] \in$ $\mathbb{R}^{1 \times K}$ and the system input matrix is given by $B \in \mathbb{R}^{K \times P}, B$ projects the envelope noise $w_{i} \in \mathbb{R}^{P}$ into $x_{i}, \psi_{i}$ is supposed to be known at each $i$ and such that noise $v_{i}$ is stationary. Namely, by $\psi_{i}=0$, noise $v_{i}$ becomes white Gaussian, as required by optimal estimators.

Because the EMG envelope noise $w_{i}$ is generally unknown, we will think that it has zero mean with uncertain both the statistics and distribution. However, to run the KF, we will consider $w_{i}$ as zero mean and white Gaussian, $w_{i} \sim$ $\mathcal{N}(0, Q) \in \mathbb{R}^{P}$, with the covariance $E\left\{w_{i} w_{j}^{T}\right\}=Q \delta_{i-j}$, where $\delta_{i}$ is a Kroneker symbol, which has unknown entries. Noise $\xi_{i}$ is zero mean and white Gaussian, $\xi_{i} \sim \mathcal{N}\left(0, \sigma_{i}^{2}\right)$, with the variance $E\left\{\xi_{i}^{2}\right\}=R=\sigma_{\xi}^{2}$ and the property $E\left\{w_{i} \xi_{j}\right\}=0$ for all $i$ and $j$.

We assume that the estimate $\hat{x}_{i} \triangleq \hat{x}_{i \mid i}$ of $x_{i}$ under the intensive nonwhite variations in the envelope will range closer to the desired envelope under the supposedly CMN. Therefore, below we will develope two possible linear approaches to shape the envelope: the KF, which requires all information about the initial values and white Gaussian noise, and the UFIR filter, which completely ignores these requirements and is thus more robust [21]. We will also consider the $H_{\infty}$ filter and call the modified solutions as $\mathrm{cKF}, \mathrm{c} H_{\infty}$ filter, and cUFIR filter.

\section{2. cKF Algorithm}

To apply the KF to (11)-(13), one can follow [21], consider a new observation $z_{i}$ as measurement differences, and write

$$
\begin{aligned}
z_{i} & =y_{i}-\psi y_{i-1}, \\
& =H x_{i}+v_{i}-\psi H x_{i-1}-\psi v_{i-1} .
\end{aligned}
$$

By taking $x_{i-1}$ from (11) and $v_{i-1}$ from (13), a new observation can be written as

$$
z_{i}=D x_{i}+\bar{v}_{i},
$$

where $D=H-\Gamma, \Gamma=\psi H F^{-1}$, and

$$
\bar{v}_{n}=\Gamma B w_{i}+\xi_{i}
$$

is white Gaussian scalar noise with the properties,

$$
\begin{aligned}
E\left\{\bar{v}_{i}^{2}\right\} & =\Gamma \Phi+R=\Gamma \Phi+\sigma_{\xi}^{2}, \\
E\left\{\bar{v}_{n} w_{i}^{T}\right\} & =\Gamma B Q
\end{aligned}
$$

where the weighted matrix $Q$ is

$$
\Phi=B Q B^{T} \Gamma^{T} .
$$

The modified state-space model (11) and (15) has now timecorrelated and white $w_{i}$ and $\bar{v}_{i}$ and the KF can be applied, if to derive the optimal bias correction gain taking into account 
the correlation. For given $y_{i}, \hat{x}_{0}, P_{0}, Q, R, \psi$, and CMN, the cKF algorithm becomes

$$
\begin{aligned}
z_{i} & =y_{i}-\psi y_{i-1} \\
P_{i}^{-} & =F P_{i-1} F^{T}+B Q B^{T}, \\
S_{i} & =D P_{i}^{-} D^{T}+R+H \Phi+\Phi^{T} D^{T} \\
K_{i} & =\left(P_{i}^{-} D^{T}+\Phi\right) S_{i}^{-1} \\
\hat{x}_{i}^{-} & =F \hat{x}_{i-1} \\
\hat{x}_{i} & =\hat{x}_{i}^{-}+K_{i}\left(z_{i}-D \hat{x}_{i}^{-}\right) \\
P_{i} & =\left(I-K_{i} D\right) P_{i}^{-}-K_{i} \Phi^{T}
\end{aligned}
$$

and, by $\psi=0$ and $\Phi=0$, it becomes the standard KF.

\section{3. $\mathrm{cH} \infty$ Algorithm}

The $H_{\infty}$ filter has been derived based on the game theory in [20] and represented in [21] as

$$
\begin{aligned}
\bar{P}_{i} & =\bar{P}_{i}^{-}\left(I \theta \bar{S}_{i} \bar{P}_{i}^{-}+H^{T} \bar{R}^{-1} H \bar{P}_{i}^{-}\right)^{-1}, \\
K_{i}^{\infty} & =\bar{P}_{i} H^{T} \bar{R}^{-1} \\
\hat{x}_{i} & =F \hat{x}_{i-1}+K_{i}^{\infty}\left(y_{i}-H F \hat{x}_{i-1}\right), \\
P_{i+1}^{-} & =F \bar{P}_{i} F^{T}+\bar{Q}
\end{aligned}
$$

where the symmetric positive definite matrices $\bar{P}_{0}, \bar{Q}$, and $\bar{R}$ must be chosen by the designer to have different meanings than in the $\mathrm{KF}$ and $\bar{P}_{1}^{-}$can be computed as $\bar{P}_{1}^{-}=F P_{0} F^{T}+Q$ [21]. Matrix $\bar{S}_{i}$ is constrained by a positive definite matrix $\left(\bar{P}_{i}^{-}\right)^{-1}-\theta S_{i}+H^{T} \bar{R}^{-1} H>0$ to keep (27) positive definite. To weight all error components equally, one must set $S_{i}=I$. A scalar bound $\theta>0$ must be small enough and, for Gaussian noise with no disturbances, $\theta=0$ makes the $H_{\infty}$ filter KF. For the $\mathrm{c} H_{\infty}$ filter, a new observation can be represented in discrete-time state-space as in (14).

\section{4. cUFIR Filtering Algorithm}

Because the UFIR filter does not require any information about noise, except for the zero mean assumption, it is more suitable for EMG signals. To provide a near optimal estimate, this filter requires an averaging horizon $[m, i]$ of $N$ points, from $m=i-N+1$ to $i$, to be optimal $N_{\mathrm{opt}}$ in the MSE sense . Since $w_{i}$ and $\bar{v}_{i}$ are both zero mean and their correlation does not produce bias, then it follows that the UFIR filter can be applied directly to (11) and (15), unlike the KF.

The cUFIR algorithm operates as follows. Given $N, y_{i}$, and $\psi$, one must set $i=N-1, N, \ldots, m=i-N+1$, and $s=i-N+K$ and compute the initial values $G_{s}=$ $\left(C_{m, s}^{T} C_{m, s}\right)^{-1}$ and $\bar{x}_{s}=G_{s} C_{m, s}^{T} Y_{m, s}$ in short batch forms via $Y_{m, s}=\left[\begin{array}{lll}y_{m} & \ldots & y_{s}\end{array}\right]^{T}$ and

$$
C_{m, s}=\left[\begin{array}{c}
D F^{-(K-1)} \\
\vdots \\
D F^{-1} \\
D
\end{array}\right] .
$$

Provided the initial values at $s$, the iteratively updated values appear for $l=s+1, \ldots, n$ using the recursions

$$
\begin{aligned}
z_{l} & =y_{l}-\psi y_{l-1}, \\
G_{l} & =\left[D^{T} D+\left(F G_{l-1} F^{T}\right)^{-1}\right]^{-1}, \\
K_{l} & =G_{l} D^{T} \\
\bar{x}_{l}^{-} & =F \bar{x}_{l-1} \\
\bar{x}_{l} & =\bar{x}_{l}^{-}+K_{l}\left(z_{l}-D \bar{x}_{l}^{-}\right),
\end{aligned}
$$

and the output estimate $\hat{x}_{i}=\bar{x}_{i}$ is taken when $l=i$. It also follows that, by $\psi=0$, the cUFIR algorithm becomes the standard UFIR filter.

The error covariance of the UFIR filter can be computed approximately by the KF error covariance if to replace the Kalman gain $K_{i}$ with $G_{i} D^{T}$ [22]. That yields

$$
\begin{aligned}
P_{i}= & \left(I-G_{i} D^{T} D\right) P_{n}^{-}\left(I-G_{i} D^{T} D\right)^{T} \\
& +G_{i} D^{T}(\Gamma \Phi+R) D G_{i} \\
& -2\left(I-G_{i} D^{T} D\right) \Phi D G_{i} \\
= & P_{i}^{-}-2\left(P_{i}^{-} D^{T}+\Phi\right) D G_{i}+G_{i} D^{T} S_{i} D G_{i} \\
= & P_{i}^{-}-\left(2 P_{i}^{-} D^{T}+2 \Phi+G_{i} D^{T} S_{i}\right) D G_{i},
\end{aligned}
$$

where $P_{i}^{-}$is given by (21) and $S_{i}$ by (22). Note that the cUFIR algorithm does not require $P_{i}$, although the recursion (37) can be included to for any purposes.

\section{Algorithms Application}

In this section, we will apply the $\mathrm{cKF}, \mathrm{c} H_{\infty}$, and cUFIR algorithms to three types of movements and compare to the KF, $H_{\infty}$, and UFIR algorithms. We will consider the EMG signal envelope extracted with the rectified signal, in which case only better denoising of the envelope is required. Next, we will compare features provided by the extracted envelopes.

For all EMG data, we specify model (11)-(13) with two states, $K=2$, and matrices

$$
F=\left[\begin{array}{ll}
1 & \tau \\
0 & 1
\end{array}\right], B=\left[\begin{array}{c}
\frac{\tau^{2}}{2} \\
\tau
\end{array}\right], H=\left[\begin{array}{ll}
1 & 0
\end{array}\right] .
$$

\subsection{Smoothed EMG Envelope}

The envelope is shaped using a rectified signal as depicted in Fig. 2a and Fig.??a with "data." Since no information about noise is provided, we tune filters to produce consistent estimates with minimal variations about the desired smooth envelope and insignificant time-delays. For the UFIR filter, we experimentally measure $N_{\mathrm{opr}}=170$, which means that data are highly oversampled. To tune the KF, we suppose that the data noise has the standard deviation of $\sigma_{\xi}=50 \mu \mathrm{V}$ and set $\sigma_{w}=0.1 \mathrm{~V} / \mathrm{s}^{2}$ for the $\mathrm{KF}$ estimate to be consistent to the UFIR estimate. For the $H_{\infty}$ filter, we consider the case of $S_{n}=I$. and $\theta=1.0 e^{-6}$. We then run the filters and arrive at estimates shown in Fig. 1b and Fig. 2b. As can be seen, all filters shape the envelope much better and consistently and there is no essential time-delays, as required. Thus letting the researchers to recognize the features of interest, we notice that methods of optimal filtering can be used to provide a more efficient shaping of the EMG signal envelope than by 
the rectification. Even so, it is seen that the envelope is still corrupted by multiple excursions.

In this case, a surface EMG signal is collected from the basic movements of fingers. In Fig. 1a and Fig. 2a, we show the waveforms of such signals, which is a part of database "S14_A1_E3" observed in a time span of $(0 \ldots 0.851) \mathrm{s}$.

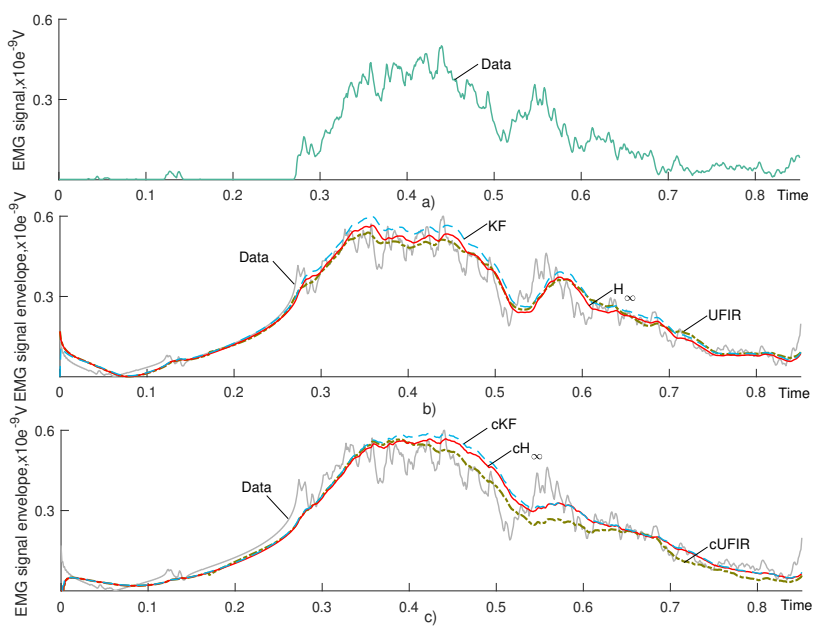

Fig. 1. A part of the EMG signal available from [25]: (a) waveform in the raw state. (b) envelope (data) obtained through the rectificed signal (solid) and its UFIR (dash-dotted), KF (bold), and $H_{\infty}$ (dashed) estimates. (c) cUFIR (dash-dotted), $\mathrm{cKF}$ (bold), and $\mathrm{c} \mathrm{H}_{\infty}$ (dashed line) estimates.

To suppress the excursions, we next tune the $\mathrm{cKF}, \mathrm{c} H_{\infty}$, and cUFIR algorithms for $\psi=0.65$ and $\bar{N}_{\text {opt }}=140$. The results are shown in Fig. 1c and Fig. 2c. Even a quick look at these figures reveals that many easily seen variations are removed from the envelope, which definitely looks more smoothed. We thus conclude that the Gauss-Markov interpretation of variations and excursions in the EMG signal envelope is useful for better envelope shaping.

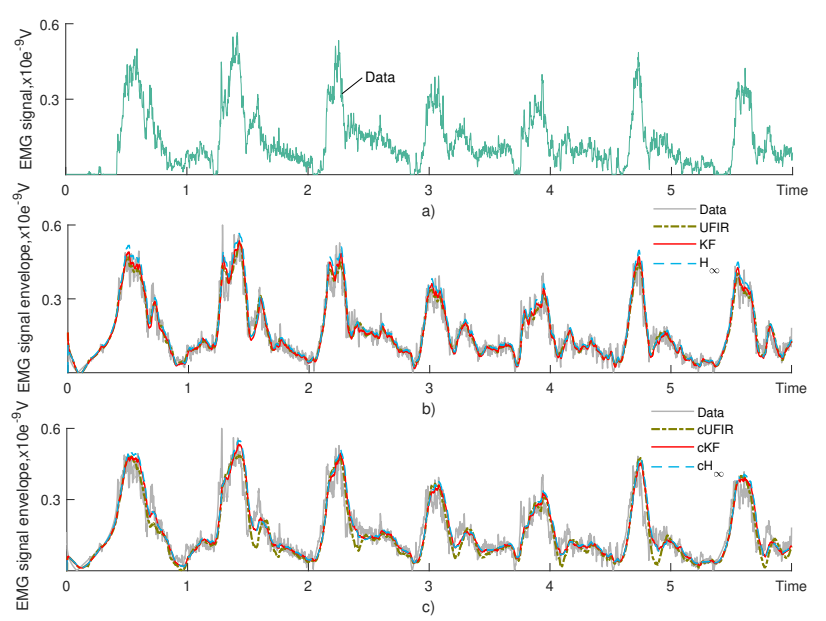

Fig. 2. A part of the EMG signal (0..6)s available from [25]: (a) waveform in the raw state. (b) envelope (data) obtained through therectificed signal (solid) and its UFIR (dash-dotted), KF (bold), and $H_{\infty}$ (dashed) estimates. (c) cUFIR (dash-dotted), cKF (bold), and $\mathrm{cH}_{\infty}$ (dashed line) estimates.
We will perform additional procedures to acquire the EMG envelope with smoother dips and peaks. For this purpose, we applied two estimators the p-shift batch optimal FIR (OFIR) estimator and Kalman filter to every estimation of the of used algorithms (KF, UFIR, $\mathrm{H}_{\infty}, \mathrm{cKF}$, cUFIR, $\mathrm{cH}_{\infty}$ ), both filters are extended for the operation of smoothing. The first of these operates as a smoother in sense $p<0$ to $|p|-\operatorname{lag}$ [22]. In the second case of KF smoothing, it used future measurements to obtain the state estimate. We used the Rauch-Tung-Striebel (RTS) smoother, which is a fixed-interval smoother. This algorithm is showed in [26].
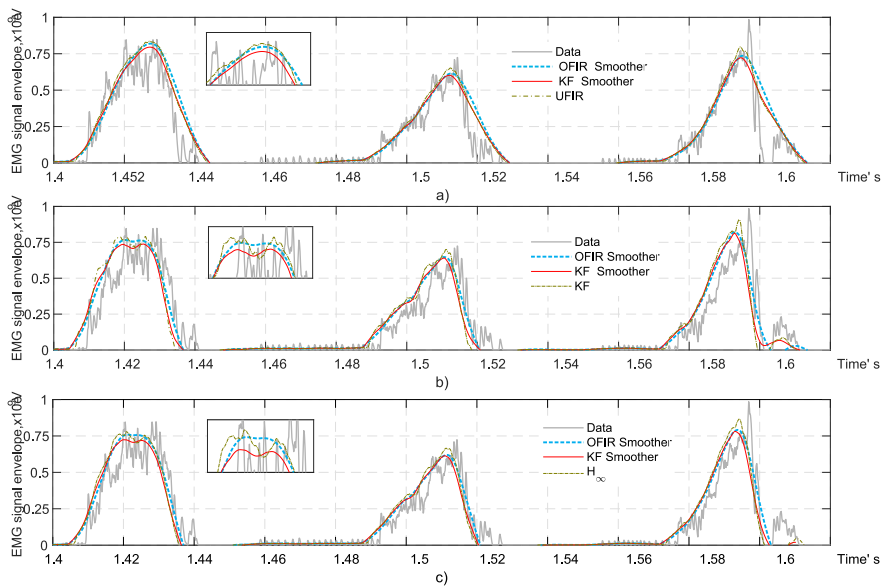

Fig. 3. A part of the EMG signal (1.4...1.6)s available from [13]: Envelope (data) obtained through the Hilbert transform (solid), OFIR Smoother (bold), KF Smoother (dash-dash), a) UFIR (solid), b) KF (Solid), and c) $\mathrm{H}_{\infty}$ (Solid) stimates.

From Fig. 3 and Fig. 4, the following can be noted. The smooth estimates of each of the algorithms. For optimum smooth we used $N_{\text {opt }}=140$ and $p=-10$.
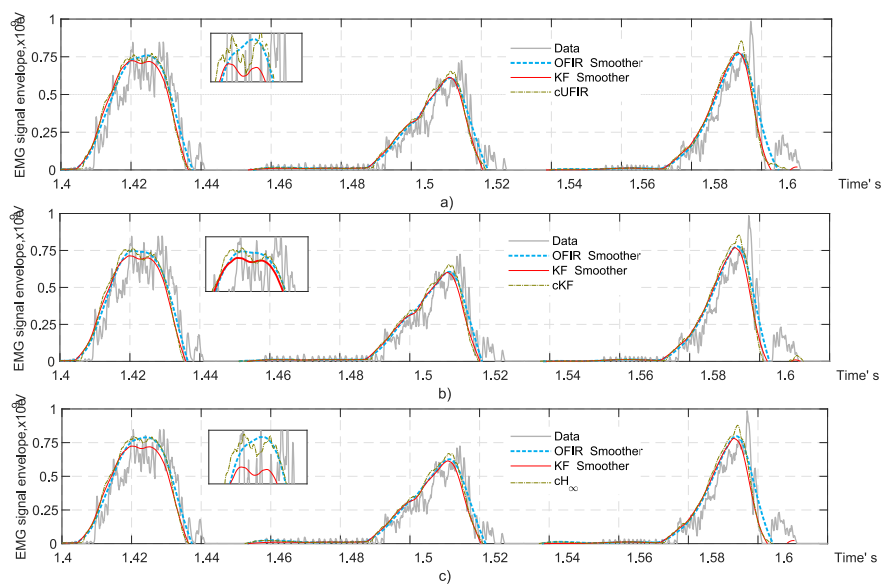

Fig. 4. A part of the EMG signal (1.4..1.6)s available from [13]: Envelope (data) obtained through the Hilbert transform (solid), OFIR Smoother (bold), KF Smoother (dash-dash), a) cUFIR (solid), b) cKF (Solid), and c) $\mathrm{cH}_{\infty}$ (Solid) stimates. 


\subsection{Test Gaussian Pulse}

Previous research has suggested that the EMG envelope must behave in a manner (distribution) by the activity of the signal, we know that given its power spectrum of a single motor unit firing with a mean rate of 15 spikes per second has a Gaussian envelope [23]. In this process, a Gaussian-shaped is present in EMG envelope distribution, where the parameters of interest are time, duration, and amplitude of the phases of activity. Gaussian is not the only shape of the EMG signal envelope to applications [24].
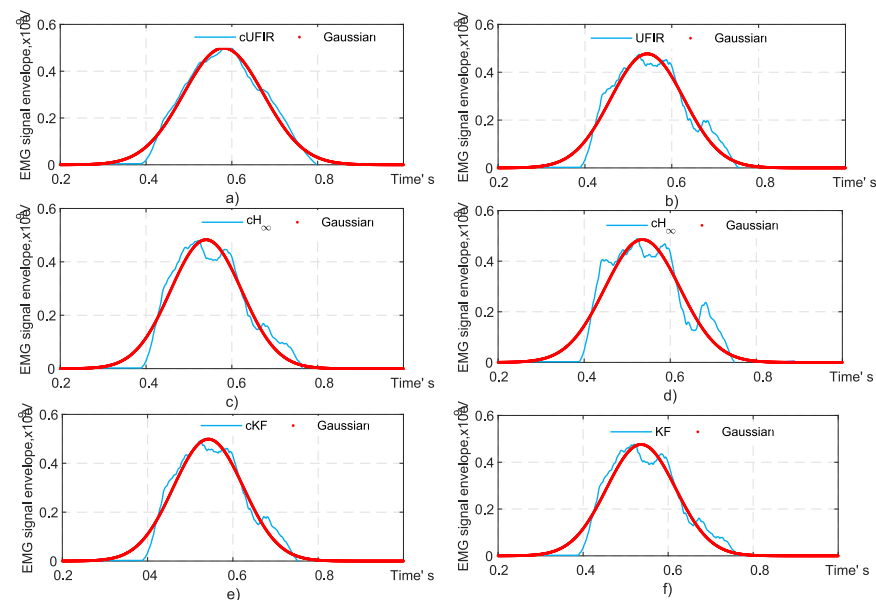

Fig. 5. Testing the envelope extracted using the algorithms by the gaussian in the MSE sense. Envelope extracted using (a) cUFIR, (b) UFIR, (c) $\mathrm{cH}_{\infty}$, (d) $\mathrm{H}_{\infty}$, (e) $\mathrm{KF}$, (f) and (e) $\mathrm{cKF}$

The EMG envelope given by each estimator has an approximation to the Gaussian pulse very significant. In Fig. 5 and Fig. 6 the Gaussian pulse is positioned to provide the best MSE sense adjustment for each algorithm in the "S1_A1_E1" signal.
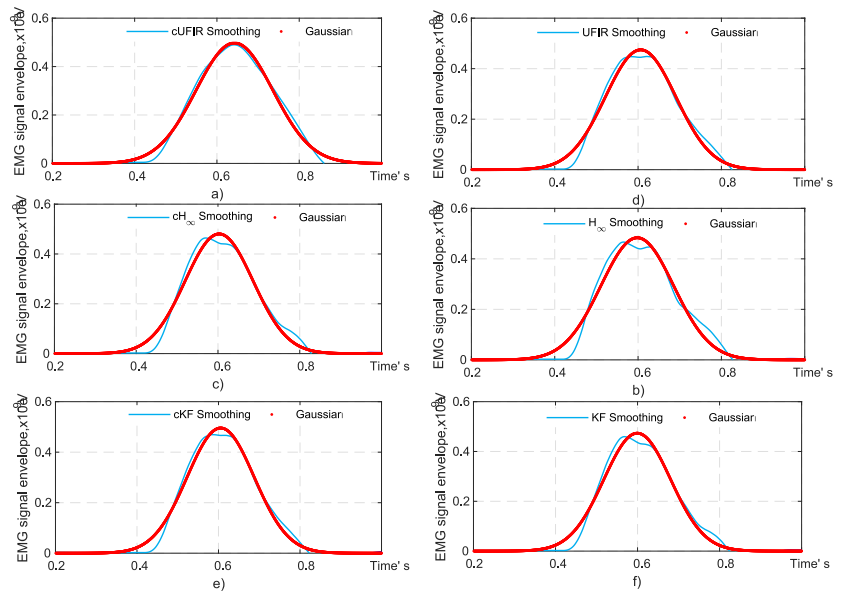

Fig. 6. Testing the estimation extracted using the algorithms by the gaussian in the MSE sense: (a) cUFIR estimation, (b) UFIR estimation, (c) $\mathrm{cH}_{\infty}$ estimation, (d) $\mathrm{H}_{\infty}$ estimation, (e) $\mathrm{cKF}$ estimation, and (f) $\mathrm{KF}$ estimation are smoothed by the use of OFIR smoothing.
TABLE I

Test Gaussian Pulse

\begin{tabular}{llll}
\hline \multicolumn{3}{c}{ RMSE } \\
\hline & Estimating & OFIR Smoothing & KF Smoothing \\
\hline KF & 0.03149 & 0.02684 & 0.2413 \\
UFIR & 0.0313 & 0.02199 & 0.02388 \\
$\mathrm{H}_{\infty}$ & 0.04068 & 0.022463 & 0.02906 \\
$\mathrm{cKF}$ & 0.0276 & 0.0198 & 0.02109 \\
$\mathrm{cUFIR}$ & 0.02623 & 0.01968 & 0.0212 \\
$\mathrm{cH}$ & 0.2885 & 0.02184 & 0.02214 \\
\hline
\end{tabular}

The table I displays the accuracy of the estimation of each algorithm for calculating a Gaussian continuous distribution function. It is observed data before estimation updating and data after treatment. It can note that envelope smoothing generated the best fit for Gaussian approximation of the signal.

\section{Control Results}

We used the muscular contraccions of the wrist to to achieve the control of $\Phi_{2}$, the elbow signal to represent $\Phi_{1}$, and the basic movements of the fingers to move the Creeper. Given the initial conditions, we integrate and find the positions of the joints by entering the values obtained from the characterization of the EMG envelope. Given $\dot{\Phi}$, we can observe the robot's trajectories to $\Phi_{1}=30^{\circ}, \Phi_{2}=120^{\circ}$, and the Creeper is forced to $0^{\circ}$, that creates the desired movement .

The objective is to get the minimum accumulated error and a minimum variation of angles in a specific time, given the joints position from an initial position to a desired position. The controller gives sudden speed changes to all diagnostics that occur during the test sequence. On this section can see the results of the speeds of joint 1 to $\Phi_{1}=30^{\circ}$ and the speed of joint 2 to $\Phi_{2}=90^{\circ}$, where, $\Phi_{2}$ has negative values. Therefore the angle variation of a joint between an instant of time and a time prior can be too wide.

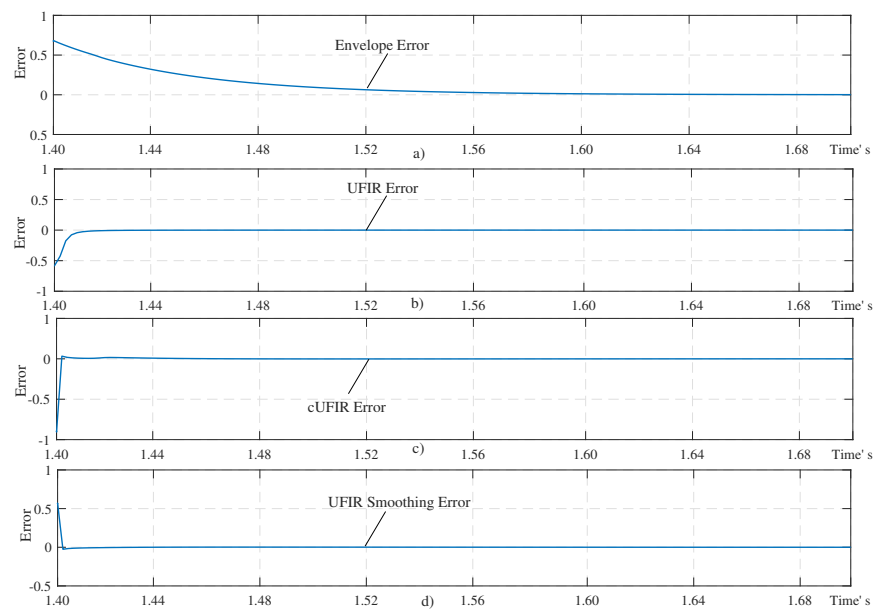

Fig. 7. Joint speeds: $\Phi_{1}$ speed is given by the solid line and $\Phi_{2}$ speed is given by the dash-dash.

The system error in response to the reference inputs for the estimate is given in Fig. 7. The benchmark targets were achieved by using PD control, we used values $k_{v}>0$ and 
$K_{p}>0$, for better stability of the system. Where $k_{v}$ is the differential constant and $K_{p}$ is the proportionality constant. The PD controller was implemented for the specified time to find rms error and thus obtain the cost function to improve the response of this controller and achieve better fit. It is noticeable that the error of the unfiltered envelope is quite high and it takes longer to achieve stability, due to EMG envelope raw produces a fast variation, such variations can then be observed in Fig.1 and Fig.2 . Respect to the results obtained, The KF, $\mathrm{H}_{\infty}$ filters had similar results to the UFIR, while $\mathrm{cKF}, \mathrm{cH}_{\infty}$ showed a behavior given by the cUFIR error. It is fairly obvious that the tripping error given in the cUFIR is large. However, the error reaches zero in a shorter space of time than in the UFIR filter, No matter that in the second the tripping error is less. Finally, we can see that the zero error is given much faster in the smoothed estimation. Trajectory tracking is not perfect, however, in regards to performance it does very well.

\section{Conclusions}

Applications of the modified cKF, $\mathrm{c} H_{\infty}$ filter, and cUFIR filter to the envelope extraction from the EMG signal data have shown their efficiency both in the envelope shaping and outliers detection. Better results were achieved by interpreting variations in the EMG signal envelope as Markov-Gauss process. Accordingly, it has been shown that the envelope extracted using the modified filters is much better smoothed and many artifacts can be removed from the EMG signal with high accuracy under the CMN assumption. Experimental verification provided based on different EMG signals have shown that the $\mathrm{cKF}, \mathrm{cH}_{\infty}$, and cUFIR algorithms are efficient when the MUAP density is low, in which case intensive excursions in the envelope are reminiscent of the colored noise. Shaped with high a MUAP density, the envelope typically demonstrates smaller variations and the filters designed become less efficient. In both cases, the color factor must be optimized to approach the desired envelope in the best way. The high variation could cause physical harm and instability in the system, this is where the theory of control is applied.Now, we may say that in the time of the estimation of the filters for their establishment is fast and the error in stationary state can be almost non-existent.

\section{HHHQFH}

[1] Orosco, E., Gaia Amoros, J., Gimenez, J., Soria, C. (2019). Deep learning-based classification using Cumulants and Bispectrum of EMG signals. IEEE Latin America Transactions, 17(12), 1946-1953.

[2] Lu, G., Brittain, J., Holland, P., Yianni, J., Green, A., Stein, J. et al. (2009). Removing ECG noise from surface EMG signals using adaptive filtering. Neuroscience Letters, 462(1), 14-19.

[3] S. Marquez-Figueroa, Y. S. Shmaliy, and O. Ibarra-Manzano, "Optimal extraction of EMG signal envelope and artifacts removal assuming colored measurement noise, Biomed. Signal Process. Contr., vol. 57, 101679, pp. $1-8$, Mar. 2020

[4] Choi, S., Han, S., Jung, D., Hwang, H., Lim, C., Bae, S. et al. Highly conductive, stretchable and biocompatible Ag-Au core-sheath nanowire composite for wearable and implantable bioelectronics.

[5] Germany - Hydrogel matrix for three enzyme entrapment. (1997). Biosensors And Bioelectronics, 12(1), xiii-xiv.
[6] Han, L., Lu, X., Wang, M., Gan, D., Deng, W., Wang, K. et al. (2016). A Mussel-Inspired Conductive, Self-Adhesive, and Self-Healable Tough Hydrogel as Cell Stimulators and Implantable Bioelectronics. Small, $13(2), 1601916$.

[7] Hasan, H., Safdar, M. H., Zahid, S., Bibi, M., Gul, A. (2018). Robotics and High-Throughput Techniques. Omics Technologies and Bio-Engineering, 155-166.

[8] T. D'Alessio and S. Conforto, "Extraction of the envelope from surface EMG signals," IEEE Eng. Med. Biol. Mag., vol. 20, no. 6, pp. 55-61, 2001.

[9] L. Chen and H. Yaru, "Feature extraction and classification of ehgbetween pregnancy and labour group using hilbert-huang transform andextreme learning machine," Computational and Mathematical Methodsin Medicine, vol. 2017, pp. 1-9, 022017.

[10] R. Kleissen and G. Zilvold, "Estimation uncertainty in ensemble averagesurface emg profiles during gait," J. Electromyography Kinesiology, vol. 4 , pp. 83-94, 1994

[11] I. Stirn, T. Jarm, Tomaz, V. Peter Kapus, V. Strojnik, "Evaluation of mean power spectral frequency of EMG signal during 100 metre crawl," Europ. J. Sport Science, vol.13, pp. 1-10, 2011.

[12] Y. Chien Hung, H. Wen Vincent Young, C.Yen Wang, Y. HungWang, P. Lei Lee, J. Horng Kang, and M. Tzung Lo, "Quantifying spasticity with limited swinging cycles using pendulum test based onphase amplitude coupling," IEEE Trans. Nucl. Sci, vol. 24, pp. 1-1, 2016.

[13] Atzori M., Gijsberts A., Heynen S., Mittaz Hager A.-G., Deriaz O., Van der Smagt P., Castellini C., Caputo B., and Müller H. 2012 IEEE International Conference on Biomedical Robotics and Biomechatronics (BioRob 2012).

[14] D. Preston and B. Shapiro, "Needle electromyography", Neurologic Clinics, vol. 20, no. 2, pp. 361-396, 2002.

[15] Aminoff, M. (1987). Electromyography in clinical practice. Churchill Livingstone.

[16] A. Guyton, Tratado de fisiologia medica. Madrid: Interamericana, 1982.

[17] Furui, A., Eto, S., Nakagaki, K., Shimada, K., Nakamura, G., Masuda, A., ... Tsuji, T. (2019). A myoelectric prosthetic hand with muscle synergy-based motion determination and impedance model-based biomimetic control. Science Robotics, 4(31), eaaw6339.

[18] Y. S. Shmaliy, "Unbiased FIR filtering of discrete time polynomial state space models," IEEE Trans. on Signal Process., vol. 57, no. 4, pp. 12411249, 2009.

[19] S. Marquez-Figueroa, Y. S. Shmaliy, and O. Ibarra-Manzano, "Optimal extraction of EMG signal envelope and artifacts removal assuming colored measurement noise," Biomed. Signal Process. Control, vol. 57 (to be published)

[20] Simon, D., Optimal State Estimaiton: Kalman, $H_{\infty}$, and Nonlinear Approaches, John Wiley \& Sons, Hoboken, NJ, 2006

[21] Y. S. Shmaliy, F. Lehmann, S. Zhao, and C. K. Ahn, "Comparing robustness of the kalman, $H_{\infty}$, and UFIR filters," IEEE Trans. Signal Process, vol. 66, no. 13, pp. 3447-3458, 2018.

[22] Y. Shmaliy, "An iterative kalman-like algorithm ignoring noise and initialconditions," IEEE Trans. Signal Process., vol. 59, pp. 2465-2473, 2011.

[23] J.-J. Chen, R. G. Shiavi, and L.-Q. Zhang, "A quantitative and qualitative description of electromyographic linear envelopes for synergy analysis," IEEE Trans. Biomed. Eng., vol. 39, no. 1, pp. 9-18, Jan. 1992.

[24] Y. Du, H. Wang, S. Qiu, W. Yao, P. Xie and X. Chen, "An advanced adaptive control of lower limb rehabilitation ro

[25] M. Atzori, A. Gijsberts, C. Castellini, B. Caputo, H. A. Mittaz, S. Elsig, G. Giatsidis, F. Bassetto, H. Müller, Electromyography data for non-invasive naturally controlled robotic hand protheses. Scientific Data 1:140053 (2014). URL https://doi.org/10.1038/sdata.2014.53

[26] D. Simon and Y. S. Shmaliy, "Unified forms for Kalman and finite impulse response filtering and smoothing," Automatica, vol. 49, no. 6 , pp. 1892-1899, Jun. 2013.

\section{Creative Commons Attribution License 4.0 (Attribution 4.0 International, CC BY 4.0)}

\author{
This article is published under the terms of the Creative \\ Commons Attribution License 4.0 \\ https://creativecommons.org/licenses/by/4.0/deed.en_US
}

